\title{
An approach towards developments of smart COVID-19 patient's management and triaging using blockchain framework
}

\author{
Pralay Kumar Lahiri \\ Kalyani Government Engineering College \\ Riman Mandal \\ University of Kalyani \\ Sourav Banerjee ( $\nabla$ mr.sourav.banerjee@ieee.org ) \\ Kalyani Government Engineering College \\ Utpal Biswas \\ University of Kalyani
}

Method Article

Keywords: Blockchain, COVID-19, Ethereum, Smart Contract,Trace- ability,Transparency

Posted Date: September 9th, 2020

DOI: https://doi.org/10.21203/rs.3.rs-70583/v1

License: (c) (i) This work is licensed under a Creative Commons Attribution 4.0 International License.

Read Full License 


\title{
An approach towards developments of smart COVID-19 patient's management and triaging using blockchain framework
}

\author{
Pralay Kumar Lahiri • Riman Mandal • \\ Sourav Banerjee . Utpal Biswas .
}

Received: date / Accepted: date

\begin{abstract}
The explosive epidemic of the coronavirus (COVID-19) has exposed the constraints in health care systems to handle public health emergencies. It's evident that adopting innovative technologies reminiscent of blockchain will facilitate in effective designing operations and resource deployments. Within the health care sector to improve the information management system by reducing delays in regulative approvals, communication between different stakeholders of the chain with the help of blockchain technology. To ensure authenticity of the information collected from public and government agencies, blockchain based system plays an important role. This paper tends to review implementation of blockchain application and opportunities in combating the COVID-19 pandemic. To trace according information involving in recent cases, deaths and recovered cases maintaining through blockchain storage system that has been proposed and implemented blockchain system based on Ethereum smart contract. An interactive model and respective algorithm has been explained with
\end{abstract}

P. K. Lahiri

Dept. of Computer Science and Engineering,

Kalyani Government Engineering College, Kalyani, Nadia, India

E-mail: pralaykr.lahiri15@gmail.com

S. Banerjee (Corresponding Author)

Dept. of Computer Science and Engineering,

Kalyani Government Engineering College, Kalyani, Nadia, India

E-mail: mr.sourav.banerjee@ieee.org

R. Mandal

Dept. of Computer Science and Engineering, University of Kalyani, Kalyani, Nadia, India

E-mail: mandal.riman@gmail.com

U. Biswas

Dept. of Computer Science and Engineering,

University of Kalyani, Kalyani, Nadia India

E-mail: utpal0172@gmail.com 
detailed analysis on information integrity, security, transparency and traceability.

Keywords Blockchain · COVID-19 · Ethereum • Smart Contract · Traceability · Transparency

\section{Introduction}

In late 2019, there was world health emergency due to COVID-19 1]. The number of COVID-19 effected cases has reached million within three months. Besides this, number of death cases also raises quickly that enforces many countries for social distancing and lockdowns. Due to cancellation of many national and international export and import businesses it affects global economy 2,3. World Health Organization (WHO) and scientists all over the world are working together to prevent and predicting the future effects of the virus.

Every day there are huge number of positive, negative effected patients and also recovered and death cases reported by national or state governments those are available for public to track the progress of COVID-19 [1]. In other hand those data are sometime incomplete that impact on prediction and analysis. There are already different applications are present in market by researchers and technical organization that helps to track COVID-19 cases [4,5].

Blockchain is a decentralized technology provides tamper-proof data with its cryptographic encryption. Along with this data privacy and transparency have drawn the attention of different industries like finance, supply chain and healthcare sector [6]. Besides this as blockchain works on decentralized system, it takes care of data recovery if in case a single server failed to interact. That helps to maintain and make each COVID-19 effected patient data available to them who have desired permission to access.

Creating this COVID-19 blockchain platform can facilitate the ability of register sharing between health care stakeholders with vital data obtainable to them during a non-mediated and economical manner whereas making certain patient privacy and security. Most significantly, cooperation between medical examiners round the world might even be increased. Accessing a patient's record is often a time intense exercise and will delay patient care. It's going to conjointly cause poor management of those records and, within the worst case situation, misdiagnosis. Digitizing records with a blockchain will scale back these shortcomings by making a network wherever reliable data is instantly obtainable. This paper provides following contributions:

- This paper has proposed an Ethereum blockchain-based frame work to fight against COVID-19 disease outbreaks by implementing smart contract verify and append during a secure and sure distributed ledger on the blockchain network victimization sensible contracts.

- Blockchain, time stamping and distributed storage options will support and strengthen the projected system with vital features just in case of infection detection data, contagion information of COVID-19 virus in real societies.

- Testing and validation of various scenarios based on system functionalities. 


\subsection{Organization}

The remaining sections of this paper are organized as follows: Section 2 discussed about background study of COVID-19 pandemic and detail explanation of blockchain technology and its implementation on COVID-19 data management. Section 3 provides proposed data flow and methodology of the implementation. Section 4 observation and discussion of the experimental results. Section 5 describes the conclusions and the future scope of the work.

\section{Related Work}

In this section, background information related to blockchain and importance of it in combating COVID-19 pandemic has been discussed.

\subsection{COVID-19}

Coronavirus (COVID-19) belongs to Severe Acute Respiratory Syndrome (SARS) family that causes cold, respiratory illness [7. At the early stage it has been suggested that the virus transmitted from animals from food market in Wuhan [8]. Respiratory droplets through sneezing and coughing is the main issues of spreading this virus. It can be spread through direct contact with mucous membranes. Depending on different surface its infectivity and duration of activeness varies 9 . COVID-19 pandemic has been declared by World Health Organization (WHO) in March, 2020 [10. As of 28 July 2020, around 16 million infection cases are reported across a 190 countries and territories, leading around 657,462 deaths 11 . In 12 author describes patient registration process on blockchain technology where COVID-19 effected data will be available in a tamperproof manner as an immune or non-immune certificate.

\subsubsection{Symptoms}

People infected with COVID-19 suffering from discussed illness that varies in different cases. It comes in view within 14 days after virus attack. In several cases it has been observed that COVID-19 effected persons have syndromes of cold, sore throat, headache, body pain, loss of taste, diarrhea and vomiting [14]. Whether in some cases people don't have any syndrome at all. People who have serious medical disease like diabetes, heart or lung disease are at the high risk and causes complications to the existed problems for individuals [10]. Fang Jiang et.al. [13] has given brief description on COVID-19 complications, syndromes and provided available clinical features with treatment policies.

\subsubsection{Preventive Measures}

No medication or vaccination has been introduced to prevent COVID-19. Therefore people should focus on reducing the risk of spread by following 
washing hand with alcohol-based sanitizer or hand wash, keep minimum two meters distance from others, keep face protected by wearing mask and practicing not to touch face without washing hands [7,10,26]. Avoiding random touch on door locks and keeps covering sneezes and coughs. Aware people about the disease and prevention technique [15, 16.

\subsubsection{Global Impact}

Not only the global economy but also people's daily life and their health impacted by this virus. Almost half of the world's population has restricted their movement as many countries declared lockdown and stray at home orders by early April [17. Most of the business has been temporarily stopped along with market and transport system [18]. Economical health of each effected country goes down and their people are suffering the most those who have works related to transport system [19,20].

\subsubsection{Mitigation Efforts}

Government and several organizations have started mitigation effort by building several applications to track COVID-19 patients and tracing their contacts. Efficient and accurate performance of the applications could help to prevent spreading this virus 21 .

The main technologies employed in this project are: Blockchain, Ethereum, Smart Contracts, Ethereum Virtual Machine, Solidity and Hashing formula. A quick description of these technologies are as follows:

\subsection{Blockchain}

Blockchain is a data structure with chain of blocks. Blocks are connected to each other through pointer which is generating hash value. As blockchain is a decentralized system so each and every nodes separately have their own record of the blockchain as shown in Fig. 1 .

\subsubsection{Block}

A block should have minimal five parts like previous block hash, nonce as numeric value, transactional hash vales in merkle root, timestamp to store time at transaction occurred, and transaction in formations to store message or other data storage as represented in Fig. 1. To validate and link to previous blocks the header information of each block used [22].

Depending on the business requirements, blockchain could be centralized or decentralized. A private blockchain is a centralized system where a centralized authority controls the blockchain network. However sometimes it could be controlled by specific nodes or candidates rather than one organization. The benefits of blockchain technology are described below. 
- As a decentralized system, it allows the users to keep control to all their transactions.

- It less likely to fail accidentally because they maintain multiple copies at multiple nodes.

- In case of durability and attack resistance, as blockchain acts at decentralized system, it increases its ability to survive malicious attack and destroy or manipulate user information. It benefits users from scams.

- By removing third-party risks users could exchange without a third-party authentication. As here omitting third-party medium and excess costs for exchanging assets, blockchain is capable to make a drastic effect on cost reduction.

\subsubsection{Ethereum}

Founded by Vitalik Buterin that provides developer a blockchain decentralized coding platform to implement smart contracts with the help of solidity language that is written in High Level Language (HLL) and later converted to Ethereum Virtual Machine (EVM) bytecode [25].

\subsubsection{Smart Contracts}

To verify the business contract or performance of the contract it is a computer protocol. Smart contract helps to improve performance of transaction as it works without third party [25]. These transactions are immutable and traceable.

\subsubsection{Solidity}

Solidity could be a contract homeward-bound language. It's designed to focus on the Ethereum Virtual Machine 25. It's statically written language, supporting inheritance, libraries and complicated user defined sorts.

\subsubsection{Hashing Algorithm}

It plays an important role within the blockchain method and confidentiality of knowledge. It transforms associated maps an absolute length of input file price to a singular fixed-length value. The formula ought to be unidirectional perform and collision-free [27. Most popular hashing functions are SHA-256 and Keccak.

Each transaction mining generates new block $\left(B_{n}\right)$ as shown in Fig. 1 consists of previous block hash $\left(B_{n-1}\right)$, nonce value $(N)$ and hash of all transaction or messages $\left(M_{n}\right)$. The mining nodes search for a nonce value such as $H\left(H\left(M_{n}\right)\left\|H\left(B_{n-1}\right)\right\| N\right)$ less than difficulty level $(D)$ as shown in Eq. 1

$$
H\left(H\left(M_{n}\right)\left\|H\left(B_{n-1}\right)\right\| N\right) \leq D
$$


Biwen Chen et al, [22] have described in his paper that Pseudo-random permutation Function indistinguishable from a random. Let's assume mapping $F:\{0,1\}^{N} \times\{0,1\}^{\beta} \Longrightarrow\{0,1\}^{N}$. Here $\beta$ is a security parameter. It is a pseudo-random permutation if

1. Given any $G \leftarrow\{0,1\}^{\beta}$, the mapping $F$ is bisection from $\{0,1\}^{N}$ to $\{0,1\}^{N}$.

2. For any probabilistic polynomial-time adversary $P A, \mid \operatorname{Pr}\left[P A^{F a}\left(1^{N}\right)=1\right]-$ $\operatorname{Pr}\left[P A^{f}\left(1^{N}\right)=1\right] \mid<\varepsilon$, where $G \leftarrow\{0,1\}^{\beta}$, if is a random permutation on $L$-bit strings and $\varepsilon$ is negligible.

3. Given any $G \leftarrow\{0,1\}^{\beta}$ and $x \leftarrow\{0,1\}^{N}$, computing $F_{G}(x)$, there exists an efficient algorithm to compute $F_{G}(x)$.

Besides, the inverse permutation $F^{-1}:\{0,1\}^{N} \times\{0,1\}^{\beta} \Longrightarrow\{0,1\}^{N}$ is the inverse of pseudo-random permutation function $F$. If $F_{G}(x)=y$, then $F_{G}^{-1}(y)=x$. Both the Data Encryption Standard (DES) and Advance Encryption Standard (AES) are the classical instances of pseudo-random permutation function.

\subsubsection{Distributed ledger}

Blockchain records and transactions by users are stored in form of distributed ledger which includes cryptographic signature and prevent the system from data losses as it shared to each members of the network.

\subsubsection{Consensus Formula}

A consensus is used to achieve necessary agreement on a node to the network. In case of decentralized blockchain system shared storage system should be efficient, secure and real time. So that each and every transaction in the system become trustful and participated nodes accepts consensus mechanism. Proof of Work (PoW) and Federated Byzantine Agreement (FBA) consensus is popular consensus algorithm [22].

\section{Methodology}

This paper has proposed a blockchain-based system for tracking information of COVID-19 patients. Decentralized applications (DApps), smart contracts, decentralized Ethereum network has been used in the proposed system that showed in Fig. 3. The proposed framework is validation based process.

Blockchain based decentralized storage system has been proposed to store patient data from different accounts. Here ERC20Token based method has been implemented for the validation process. So that transaction of message cannot get inserted maliciously. One of the most significant tokens is called ERC-20Token, which has emerged as the technical standard used for all smart contracts on the Ethereum blockchain for token implementation. As this is a decentralized system, patient details can be traced from another account. 


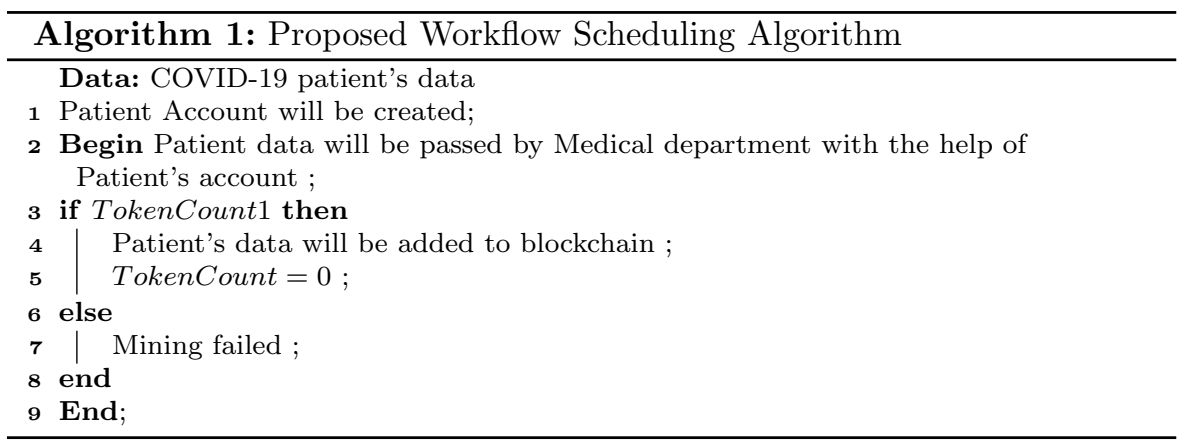

Here token has been implemented for validation from different sectors. So that insurance claimer becomes eligible or not-eligible for to claim insurance amount.

Here two different accounts have been applied to maintain patient data. Once the patient account has been created its details has to be entered by the medical department or healthcare or validator account as patient account is not able to add any details by itself. To enter any record healthcare has to pass minimum one token value. As per algorithm 1 it is clear that without minimum one value of token patient details will not be able to pass the validation process and mining will fail.

Once the patient details have been entered then available token count of the account will become zero. Next if the patient updated data need to store then again it has to follow from the step 1 to step 9 of algorithm 1 . To find patient details, it does not go through ERC20Token validation check and every patient detail can be traced from each account. If there has been any attempt to insert any malicious data, validation process will prevent it. As without minimum one token count, no one will be able to add new transaction.

\section{Observation and Discussion}

In case of develop, deploy, debug and test solidity smart contracts in Ethereum blockchain, Remix is one of the popular components. Remix has its two flavors as browser based and installable IDE. It has to go through access https: //remix.ethereum.org to develop, deploy smart contract in case of browser based one. On the other hand for installable IDE it can run and use all those features in offline based. To run it offline based it must install Nodejs on the system.

\subsection{Pre-requisites}

To develop the blockchain system here Windows 10 Pro operating system with Intel Core i5-4210U CPU @ 1.70GHz, 8.00 GB of RAM, 64-bit Operating 
System has been used. Net framework need to be installed on the system. Here it has to visit the Microsoft website and download the .Net framework and installed it on the system. Using direct window Installer Nodejs version v10.16.3 has been installed on the system. Now by using command prompt, executed npm install remix-ide-g, command to install remix-ide on the system and started remix-ide by using remix-ide command. Accessing local folder from remix-ide is available by http://localhost:8080.

\subsection{Smart contract}

Implementation of smart contract using solidity based on object oriented, highlevel language. Smart contracts are the main to control data governance in Ethereum. To create Ethereum Virtual Machine (EVM) solidity uses Python, JavaScript and $C++$ which support libraries, inheritance and other complex features which helps to create voting, crowd funding, multi signature wallets etc. When deploying contracts, system currently used solidity version 0.5.17 that works for ERC20Token.

\subsection{Storage}

As Ethereum performs on decentralized based system so it maintains records copy in each and every nodes. A ledger is a storage system where all historical transactions get stored included contracts. Once deployment of contract is done the mining get started and after a successful mining records get stored at blockchain as a new block. As block created based on timestamp so all subsequent blocks get stored at block chain in timely manner.

Trie is used as database to store accounts in blockchain which is defer from merkle tree.

Remix-ide provides an option to store transactions on json file structure so that we could use the transactions backup for future.

This paper has implemented two smart contracts named Healthcare.sol and Patients.sol. Implementation of smart contract has been done by using solidity as programming language of 0.5.17 version that supports ERC20Token. Here smart contract used with ERC20Token that helps validator to pass token as certifying from their department whether the candidate identity and other contract match.

\subsection{Dataset description}

At this stage basically the dataset is scaled in such a way that it is fit for further processing. In this step at first standardization is done such that for every attribute patientNo, patientName, patientAddress, govtIDNumber, patientAdmitDate, patientReleaseDate, patientCOVIDStatus is set as Null. 
As a result preliminary there will be no record present and system will not hamper its role.

Combine Two separate smart contract Pateints.sol and Healthcarer.sol, Patients.sol is used at Client side to insert Patient Data, COVID - 19 status of patient. Here validator is assumed as Government sectors like healthcare or medicine or hospital, etc. So we assume that here Patient Data will be Secured.

\subsection{Patients.sol}

In section 4.4 have described what are the parameter has been implemented to store a patient details. Here in this contract we applied ERC20Token concept that helps both way to store a patient details and also from validator side to check and validate it. ERC20 is nothing but a technical specification but a technology or software or code. ERC20Token is similar to HTTP protocol to define where the token should implement. In case of transferring token it maintain the balance of token. It is implemented on Ethereum based smart contract.

\subsection{Healthcare.sol}

It works for validator side to trace any patient details and also to validate by passing token as an approval. Here we have assumed to take only one government sector related to healthcare or medicine or hospital to do verification. So to add updated patient details to blockchain it has to pass through the validation and collect minimum one token.

In our system average block size is 675.08 bytes. In Fig. 4 it represents the sample of 25 blocks where minimum block size is 661 bytes and maximum block size is 690 bytes. As the proposed system is distributed in nature, it makes the system robust and durable. Each system holds its local data and shared data on distributed ledgers. As it is a distributed system so connected devices also have copy of local data. If any of the system goes down then it has multiple backups to recover it. It requires very high computation range to make changes in side any block that ensures security.

Validator as Healthcare have to pass single token so that patient account get verified first as shown in Fig. 6 then patient details have been passed to the blockchain it generate the hash value shown in Fig. 7. Now as token value reverts back to zero, patient account is unable to add any malicious date to the chain. To add new block validator have to make token value one. The updated COVID status of the patient is as shown in Fig. 8 .

As it is a decentralized system so patient and validator both can trace other patient data by using patient number. Here in Fig. 9 it shows a result of other patient details from previous patient account.

To elaborate on the importance, effectiveness and global impact, some different points have been discussed below. 
Here, blockchain has made the message system not only secure but also efficient in processing. Comparing with other healthcare system it always suffers from scalability and security issues. Implementation of blockchain with decentralized technology makes the resource available to every node whenever and wherever it needs. So, besides a secure system, it resolves the scalability.

The proposed system not restricted for healthcare system but also in many other applications like cloud document storage, secure social message passing system, bank information system, defense sectors, etc. as it works on decentralization by using blockchain technology. In all above cases it works on different sectors but the proposing system could be same.

The proposed work mainly focused on two sections, one is security and another is transparency. By following the proposed workflow it eliminates the security issues faced by healthcare system and as here decentralization with blockchain has been applied, so it also covers the transparency of the patient data as those become tamper-proof.

As this paper focused on security and transparency for storage system that makes data tamper-proof by implementing a trustful validation system. Rather than this, it could help to build a resource storing system for the banking industry and make it more secure and efficient for transaction storage and passing. So this paper provides the window to other researchers for other application-based research works on similar storage and a transaction-based system where scalability is needed.

\section{Conclusion}

In this paper, we have discussed blockchain application and opportunities in combating the COVID-19 pandemic. The proposed model is capable to trace other COVID patient details. Our proposed solution implemented Ethereum smart contracts on blockchain technology for COVID-19 that can be implemented in other healthcare systems.

The developed system would update the DApps and each patient detail will be traceable to each account. The presented system architecture, sequence diagram and algorithms can be easily generalized for tracking various infectious diseases. The proposed solution addresses the problems faced in the current pandemic crisis such as is data manipulation and single point of failure. Furthermore, it mitigates malicious activities due to its inherent cryptography security features of blockchain technology. Overall, the proposed solution is generic enough that it can be adapted to use for data collection and for future perspective could be use for report statistics on other infectious diseases including Malaria, HIV and TB etc. This is possible as blockchain encourages the sharing and reporting of data among stakeholders in a network. The proposed solution could be used streamline communication between patients and healthcare professionals. It can connect all research and healthcare communities within the same network to use and share a trusted secure database that is tamper-proof. However, it should be noted that all relevant stakeholders must 
be involved in implementing the proposed solution so that it is sustainable, efficient and trustworthy.

\section{References}

1. WHO, "Rolling updates on coronavirus disease (COVID-19)," World Health Organization, [Online]. Available: https://www. who.int/emergencies/diseases/novel-coronav irus-2019/events-as-they-happen [Accessed 25 May 2020].

2. G. Ramsay, "Japanese PM and IOC chief agree to postpone 2020 Olympics until 2021," CNN, 24 March 2020. [Online]. Available: https://edition.cnn.com/2020/03/24/sport /olympics-postponementtokyo-2020-spt-intl/index.html [Accessed 25 May 2020].

3. "BIE Member States confirm one-year postponement for Expo 2020," Expo 2020 Dubai UAE, [Online]. Available: https://www. expo2020dubai.com/en/whats-new/expo-storie s/bieannouncement [Accessed 25 May 2020]

4. P. Bischoff, "COVID-19 App Tracker: Is privacy being sacrificed in a bid to combat the virus?," CompariTech, 20 April 2020. [Online]. Available: https://www.comparitech.co $\mathrm{m} / \mathrm{blog} /$ vpnprivacy/coronavirus-apps/ [Accessed 25 May 2020].

5. K. Warner and S. A. Nowais, "Coronavirus: Doctors urge public to help track Covid19 cases with tracing app," The National, 28 April 2020. [Online]. Available: https: //www.thenational.ae/uae/health/coronavirus-doctors-urgepublic-to-help-track -covid-19-cases-with-racing-app-1.1012267 [Accessed 25 May 2020].

6. Fakhri Alam Khan, Muhammad Asif, Awais Ahmad, Mafawez Alharbi, and Hanan Aljuaid, Blockchain technology, improvement suggestions, security challenges on smart grid and its application in healthcare for sustainable development. Sustainable Cities and Society, Volume 55, April 2020, 102018.

7. B. L. Tesini, "MSD Manual," May 2020. [Online]. Available: https://www.msdmanuals .com/professional/infectiousdiseases/respiratory-viruses/coronaviruses-and-a cute-respiratorysyndromes-covid-19,-mers,-and-sars\#v47572273 [Accessed 3 June 2020].

8. G. Readfearn, "How did coronavirus start and where did it come from? Was it really Wuhan's animal market?," The Guardian, 28 April 2020. [Online]. Available: https: //www.theguardian.com/world/2020/apr/28/how-did-thecoronavirus-start-where-d id-it-come-from-how-did-it-spreadhumans-was-it-really-bats-pangolins-wuhan-a nimal-market [Accessed 3 June 2020].

9. D. J. Cennimo, "Coronavirus Disease 2019 (COVID-19)," MedScape, 1 June 2020. [Online]. Available: https://emedicine.medscape.com/article/2500114-overview\#a9 [Accessed 3 June 2020]

10. "Coronavirus disease 2019 (COVID-19)," MayoClinic, 22 May 2020. [Online]. Available: https://www.mayoclinic.org/diseasesconditions/coronavirus/symptoms-causes/sy c-20479963. [Accessed 3 June 2020].

11. "COVID-19 Dashboard by the Center for Systems Science and Engineering (CSSE)," Johns Hopkins University, 2020. [Online].Available: https://gisanddata.maps .arcgis.c om/apps/opsdashboard/index.html\#/bda7594740fd40299423467b48e9ecf6] [Accessed 3 June 2020].

12. Bansal, A., Garg, C. \& Padappayil, R.P. Optimizing the Implementation of COVID-19 "Immunity Certificates" Using Blockchain. J Med Syst 44, 140 (2020). https://doi.org/ 10.1007/s10916-020-01616-4

13. Jiang, F., Deng, L., Zhang, L. et al. Review of the Clinical Characteristics of Coronavirus Disease 2019 (COVID-19). J GEN INTERN MED 35, 1545-1549 (2020). https://doi.or $\mathrm{g} / 10.1007 / \mathrm{s} 11606-020-05762-\mathrm{w}$

14. "Coronavirus Disease 2019 (COVID-19)," CDC Center for Disease Control and Prevention, May 2020. [Online]. Available: https://www.cdc.gov/coronavirus/2019-ncov/symp tomstesting/symptoms.html. [Accessed June 2020].

15. "Coronavirus disease (COVID-19): Prevention and risks," Government of Canada, 29 May 2020. [Online]. Available: https://www.canada.ca/en/public-health/services/di seases/2019-novel-coronavirus-infection/prevention-risks.html] [Accessed 3 June 2020]. 
16. "How to Protect Yourself \& Others," CDC Center of Disease Control and Prevention, 24 April 2020. [Online]. Available: https://www.cdc.gov/coronavirus/2019-ncov/prev ent-gettingsick/prevention.html [Accessed 4 June 2020].

17. A. Sandford, "Coronavirus: Half of humanity now on lockdown as 90 countries call for confinement," Euronews, 3 April 2020. [Online]. Available: https://www.euronews.com/2 020/04/02/coronavirus-ineurope-spain-s-death-toll-hits-10-000-after-record-9 50-new-deathsin-24-hou [Accessed 4 June 2020].

18. R. Wright, "The world's largest coronavirus lockdown is having a dramatic impact on pollution in India," CNN, 1 April 2020. [Online]. Available: https://edition.cnn.com/20 20/03/31/asia/coronaviruslockdown-impact-pollution-india-intl-hnk/index.html [Accessed 4 June 2020].

19. S. P. Chico Harlan, "Italy extends coronavirus lockdown to entire country, imposing restrictions on 60 million people," Wahington Post, 10 March 2020. [Online]. Available: Chico Harlan and . [Accessed 4 June 2020].

20. R. S. Carmen M. Reinhart, "How can we prevent a COVID-19 food crisis?," World Economic Forum, May 2020. [Online]. Available: https://www.weforum.org/agenda/202 0/05/preventing-a-covid-19-food-crisis/ [Accessed 4 June 2020].

21. A. F. et. al., "Simulating SARS-CoV-2 epidemics by region-specific variables and modeling contact tracing App containment," MedRxiv, 2020.

22. Z. Zheng, S. Xie, H. Dai, X. Chen and H. Wang, "An Overview of Blockchain Technology: Architecture, Consensus, and Future Trends," in IEEE 6th International Congress on Big Data, Honolulu, 2017.

23. S. Nakamoto, Bitcoin: A Peer-to-Peer Electronic Cash SyNakamoto, S. (2008). Bitcoin: A Peerto- Peer Electronic Cash System. Consulted, 19. doi 10.1007/s10838-008-90620stem. J. Gen. Philos. Sci., vol. 39, no. 1, pp. 5367, 2008

24. B. Chen, L. Wu, H. Wang, L. Zhou and D. He, "A Blockchain-Based Searchable PublicKey Encryption With Forward and Backward Privacy for Cloud-Assisted Vehicular Social Networks," in IEEE Transactions on Vehicular Technology, vol. 69, no. 6, pp. 5813-5825, June 2020, doi: 10.1109/TVT.2019.2959383

25. Kole, Swapnil. (2020). COVID-19 Database on Consortium Blockchain. doi 10.13140/R G.2.2.28358.22088/1

26. Marbouh, Dounia \& Abbasi, Tayaba \& Maasmi, Fatema \& Omar, Ilhaam \& Debe, Mazin \& Salah, Khaled \& Jayaraman, Raja \& Ellahham, Samer. (2020). Blockchain for COVID-19: Review, Opportunities and a Trusted Tracking System. doi $10.36227 /$ techr xiv.12609344

27. Torky, Mohamed \& Hassanien, Aboul Ella. (2020). COVID-19 Blockchain Framework: Innovative Approach. doi 10.13140/RG.2.2.21696.48640 


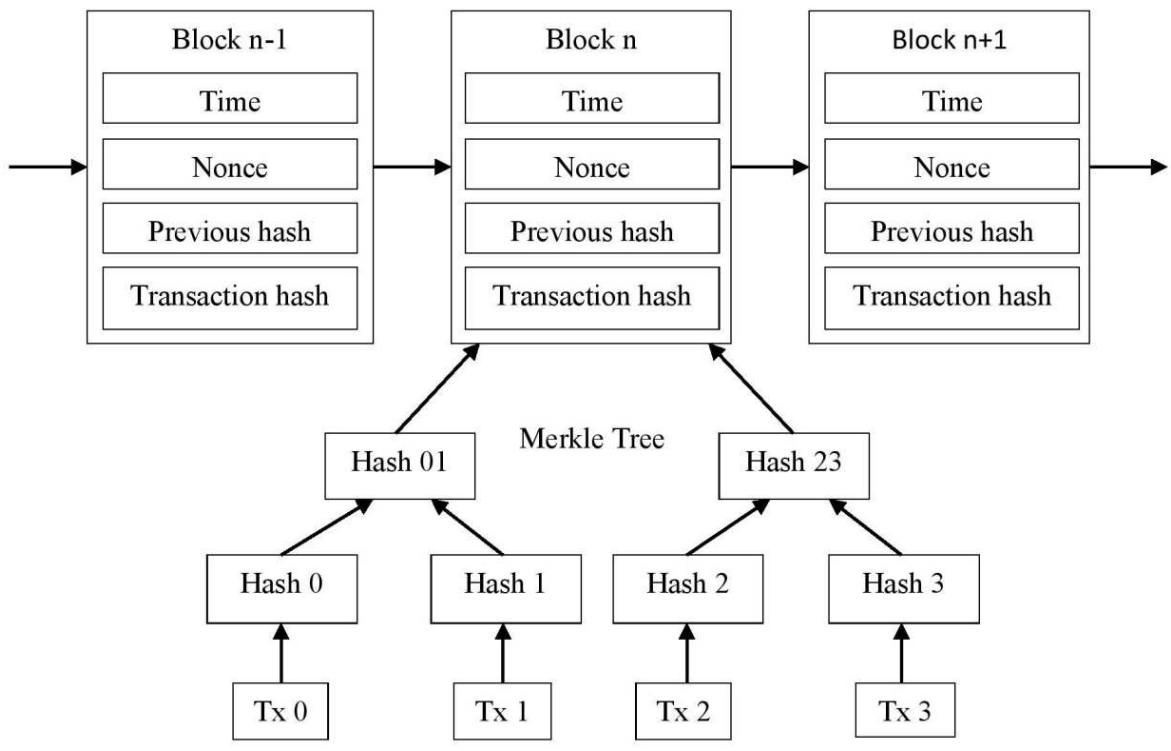

Fig. 1 Block structure in Blockchain

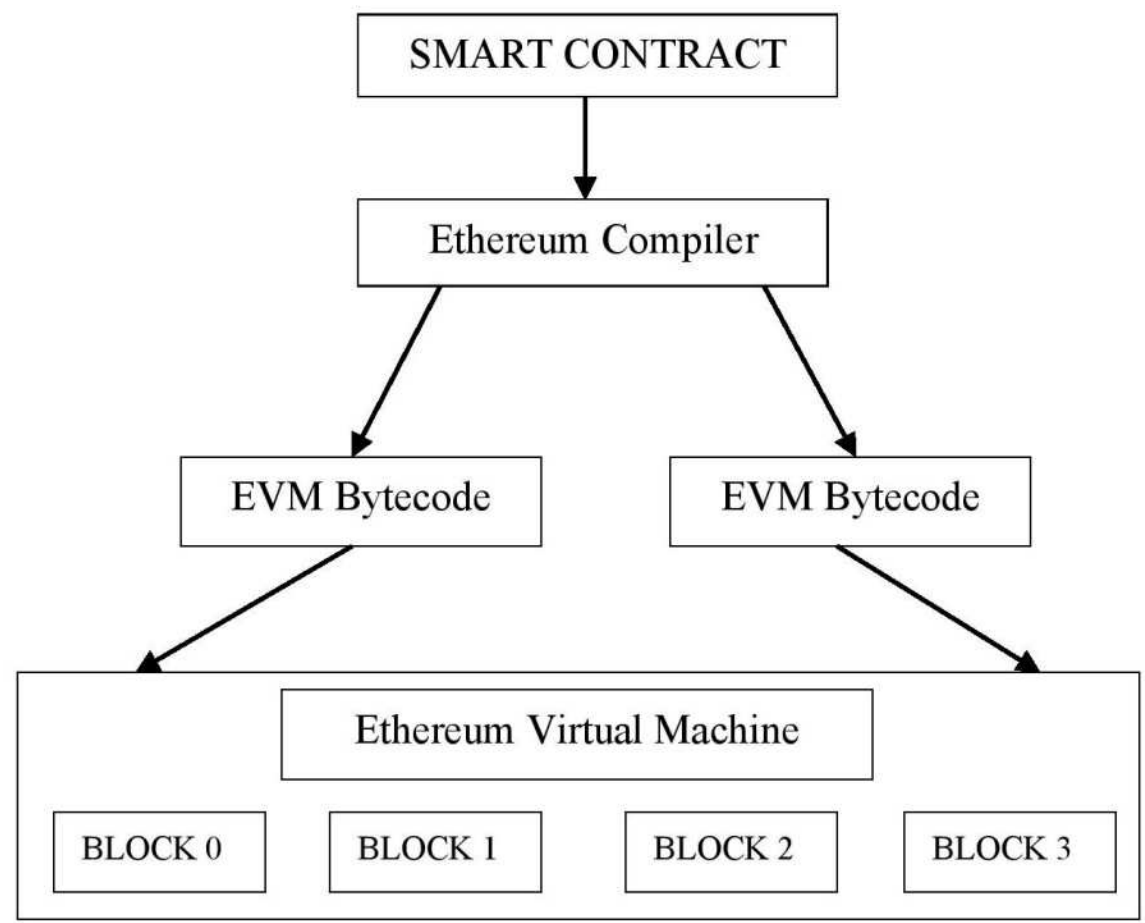

Fig. 2 Smart contract execution 


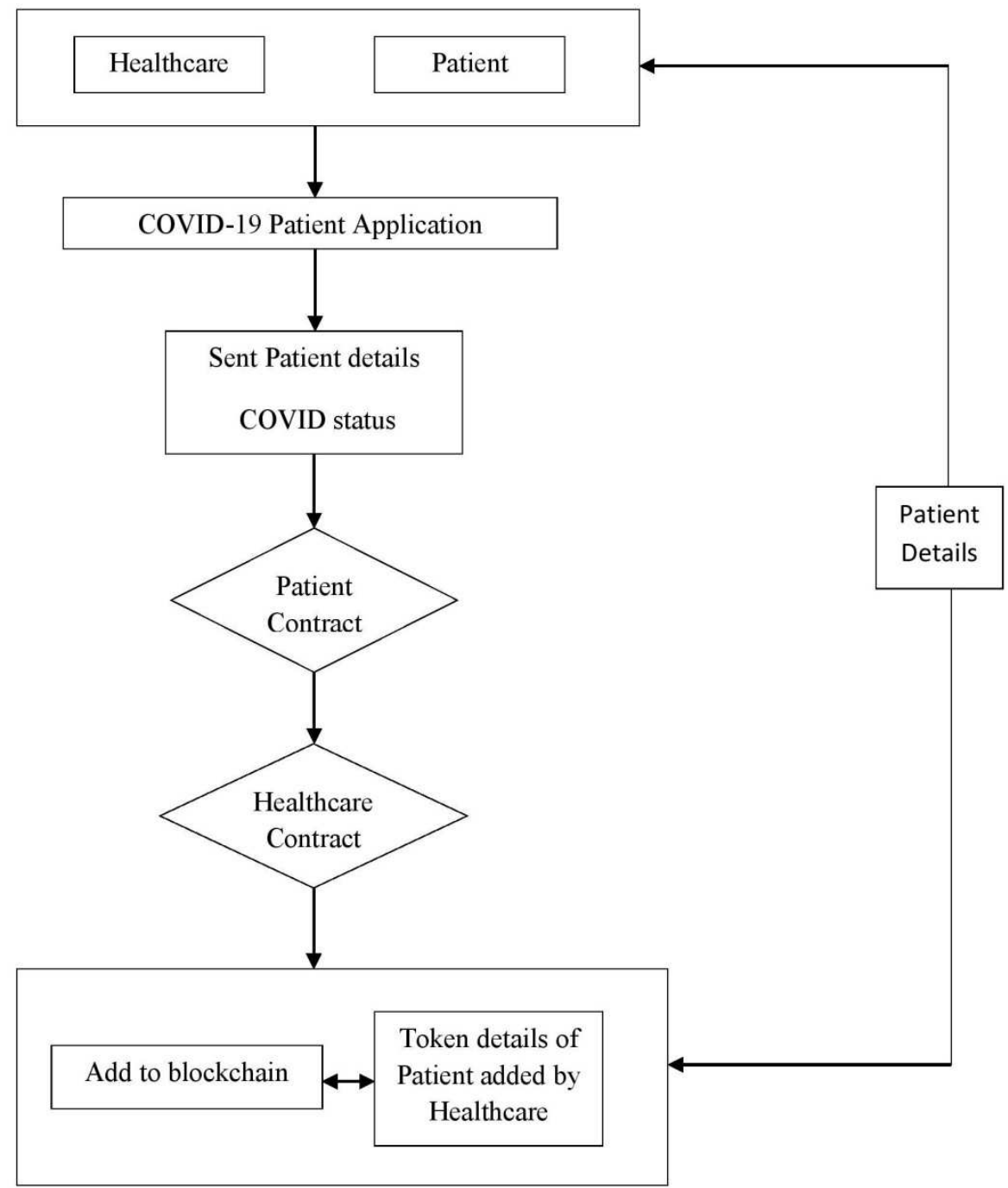

Fig. 3 Proposed Blockchain workflow 


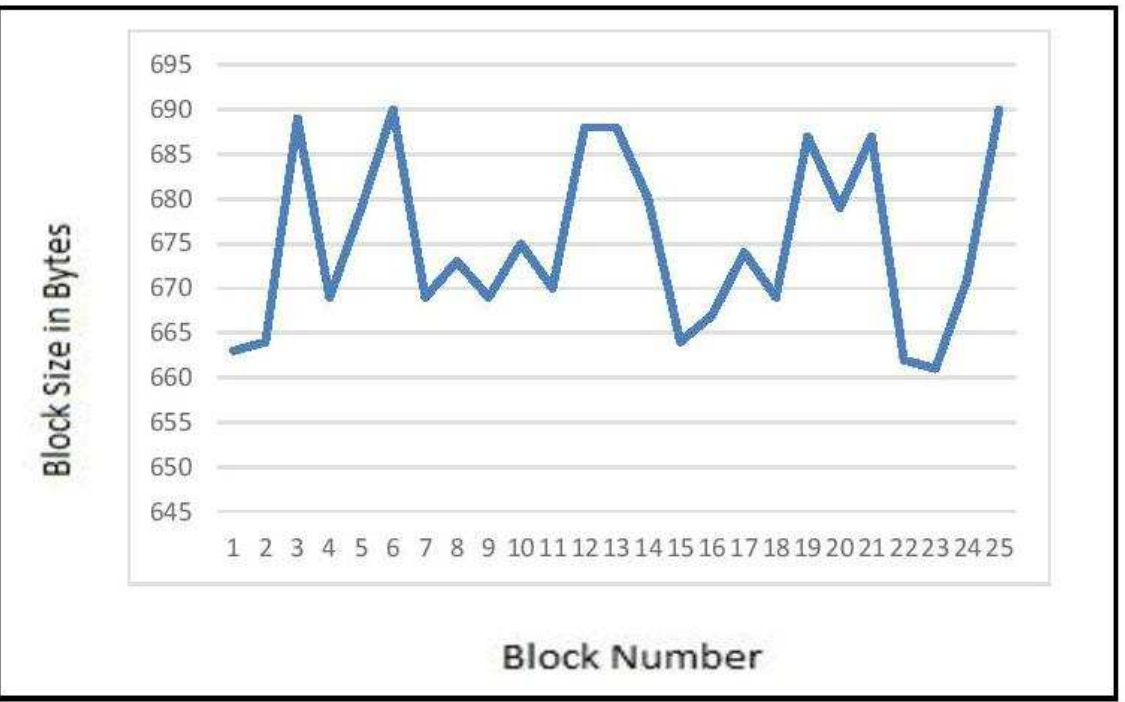

Fig. 4 Proposed Blockchain workflow

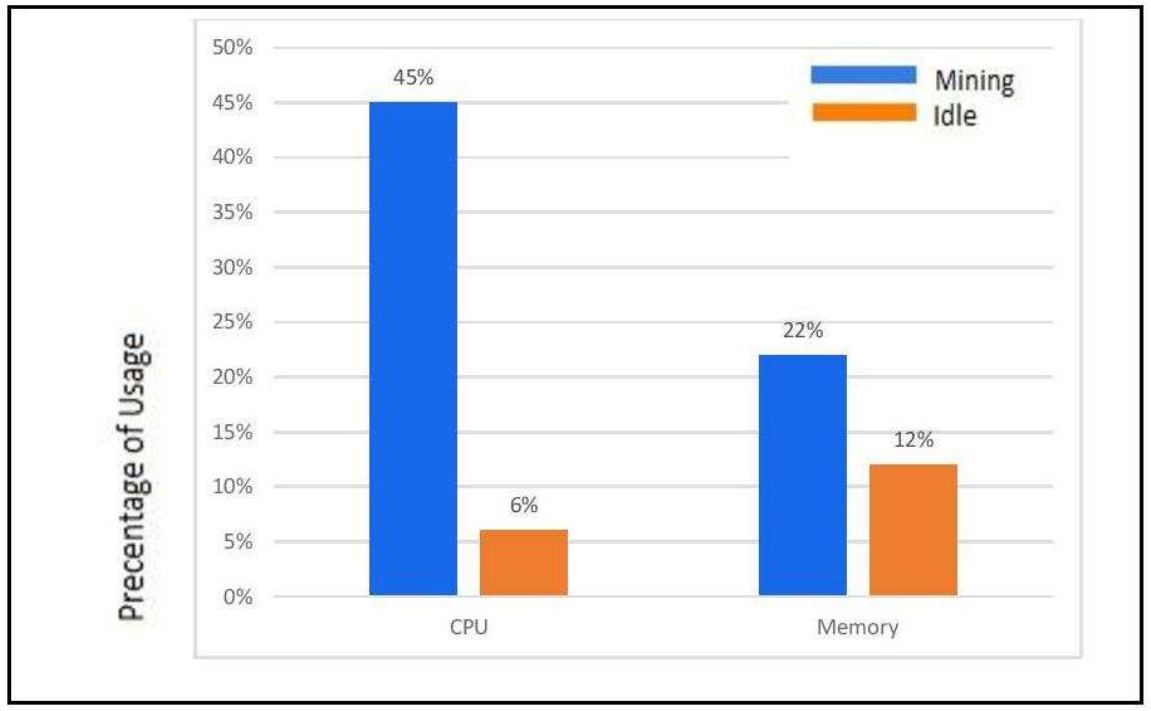

Fig. 5 Block size 


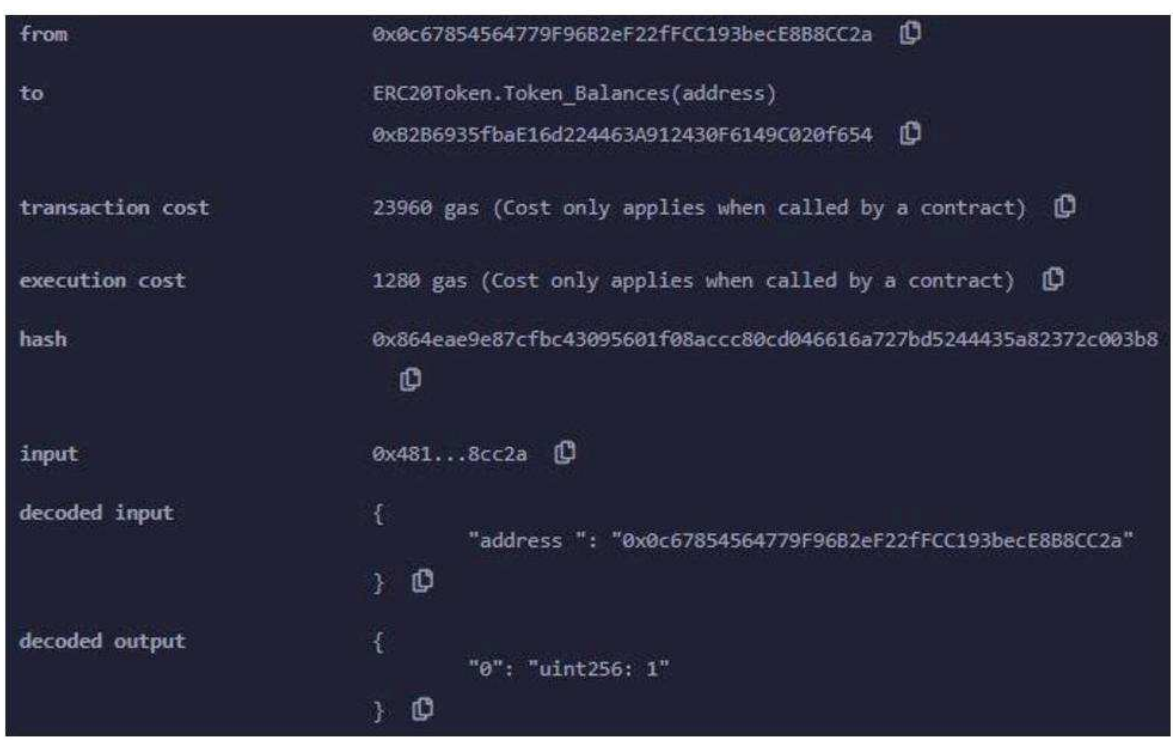

Fig. $6 \mathrm{CPU}$ and memory utilization comparison during mining and idleness 


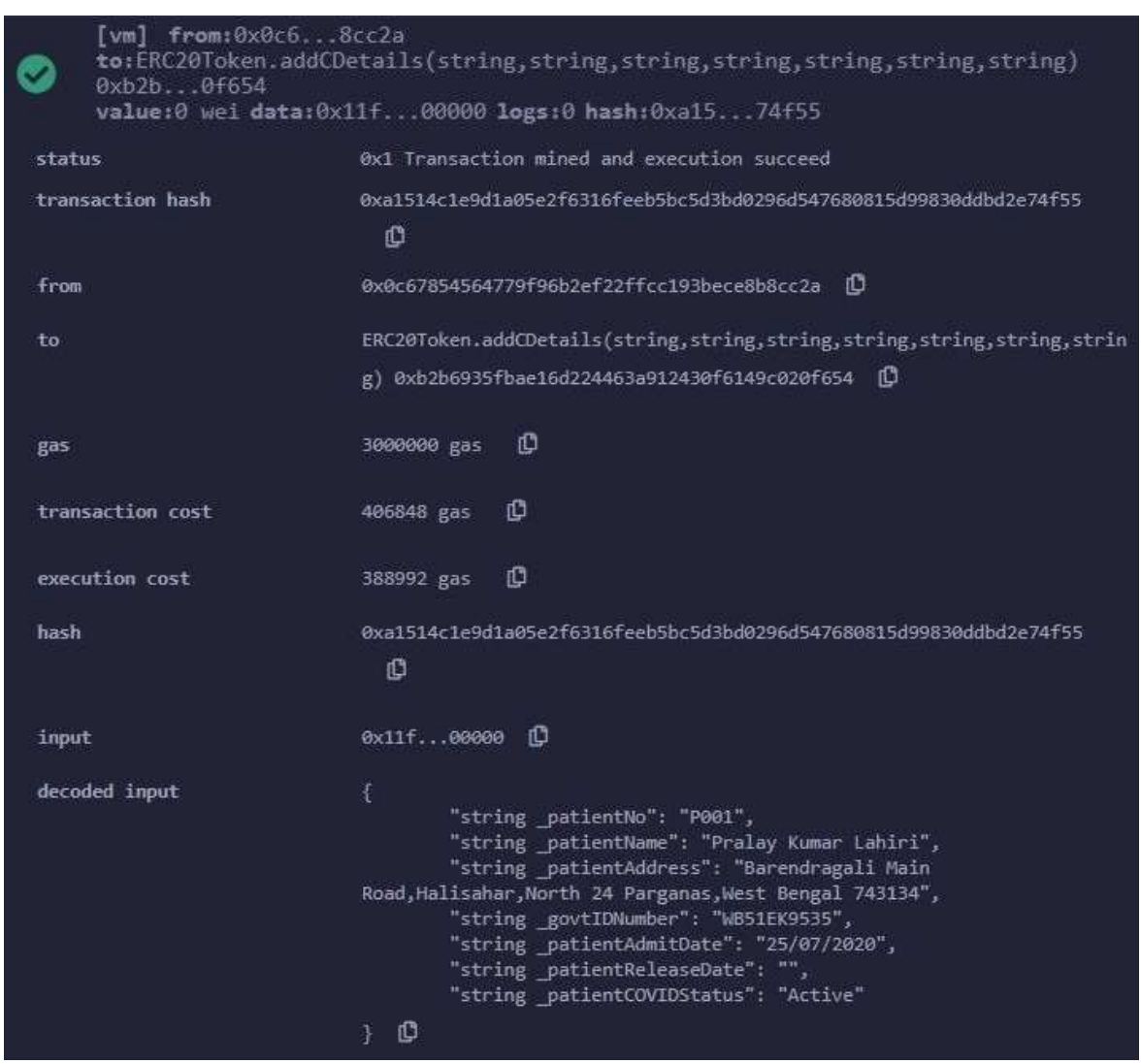

Fig. 7 Token value passed by Healthcare 


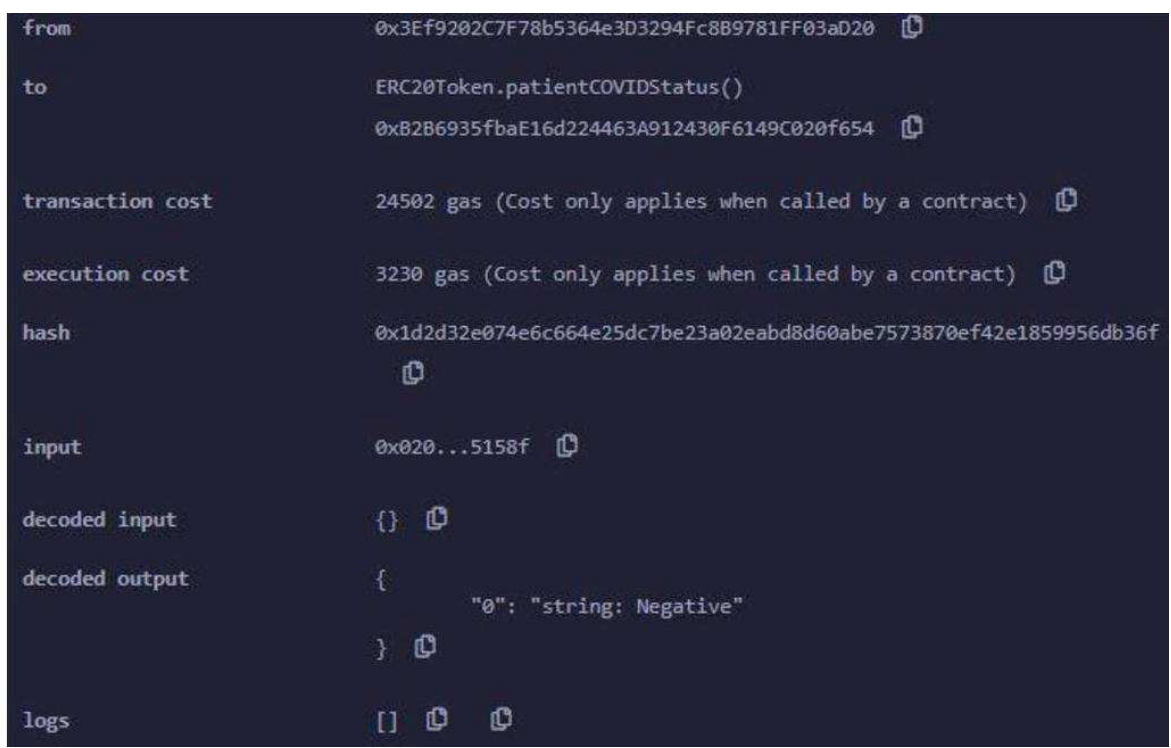

Fig. 8 Patient data inserted successfully

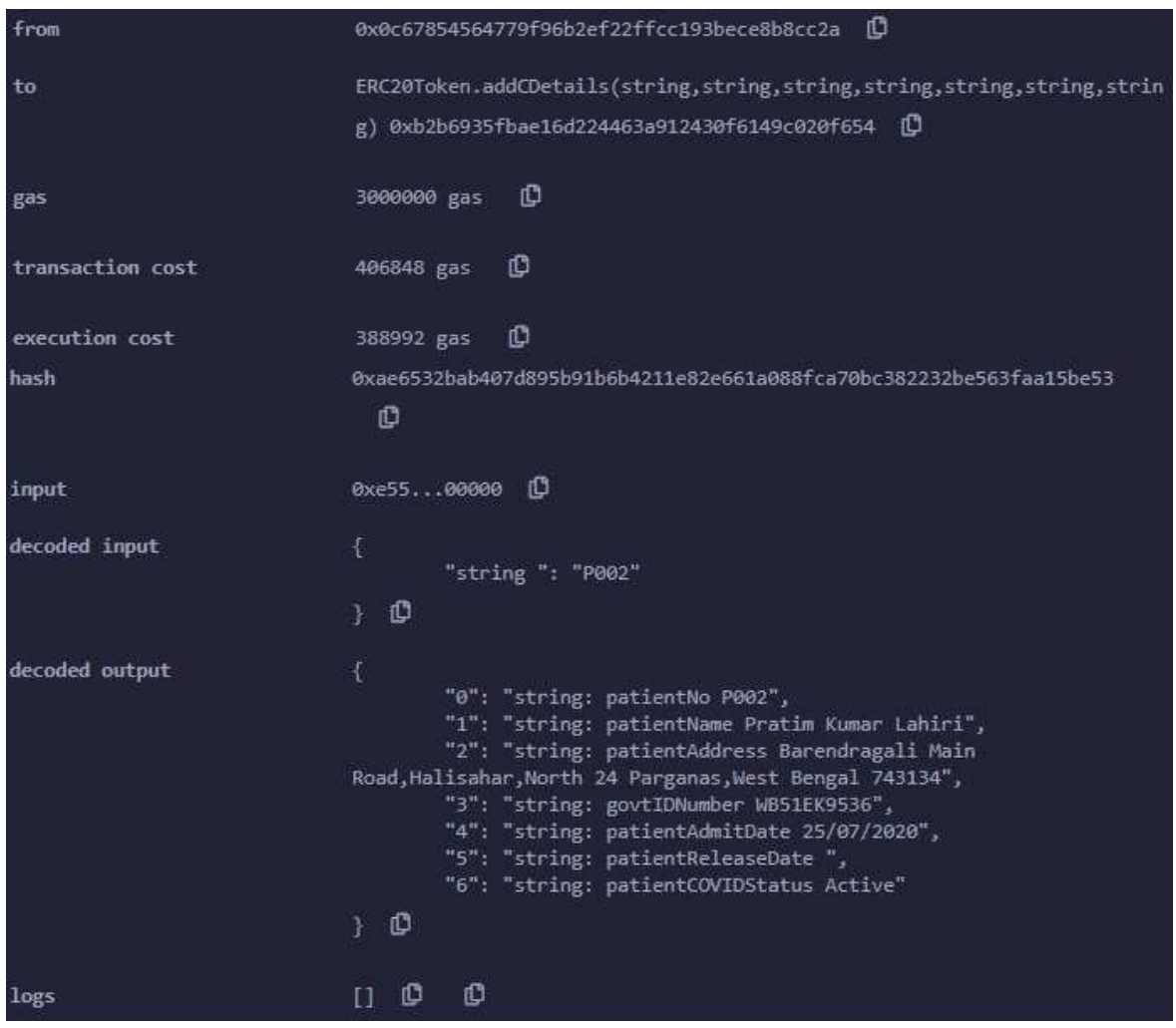

Fig. 9 Updated COVID status of patient 
Figures

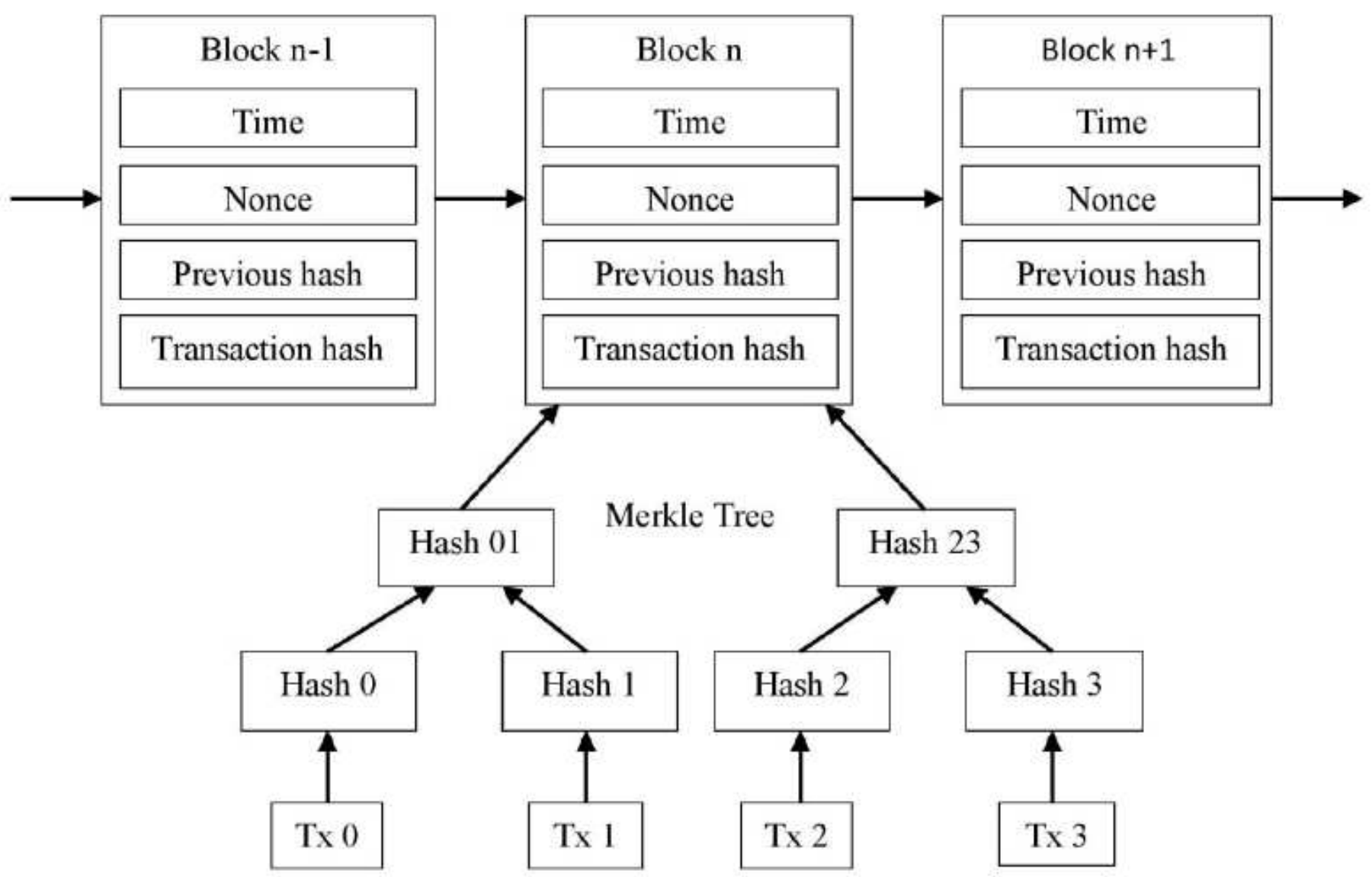

Figure 1

Block structure in Blockchain 


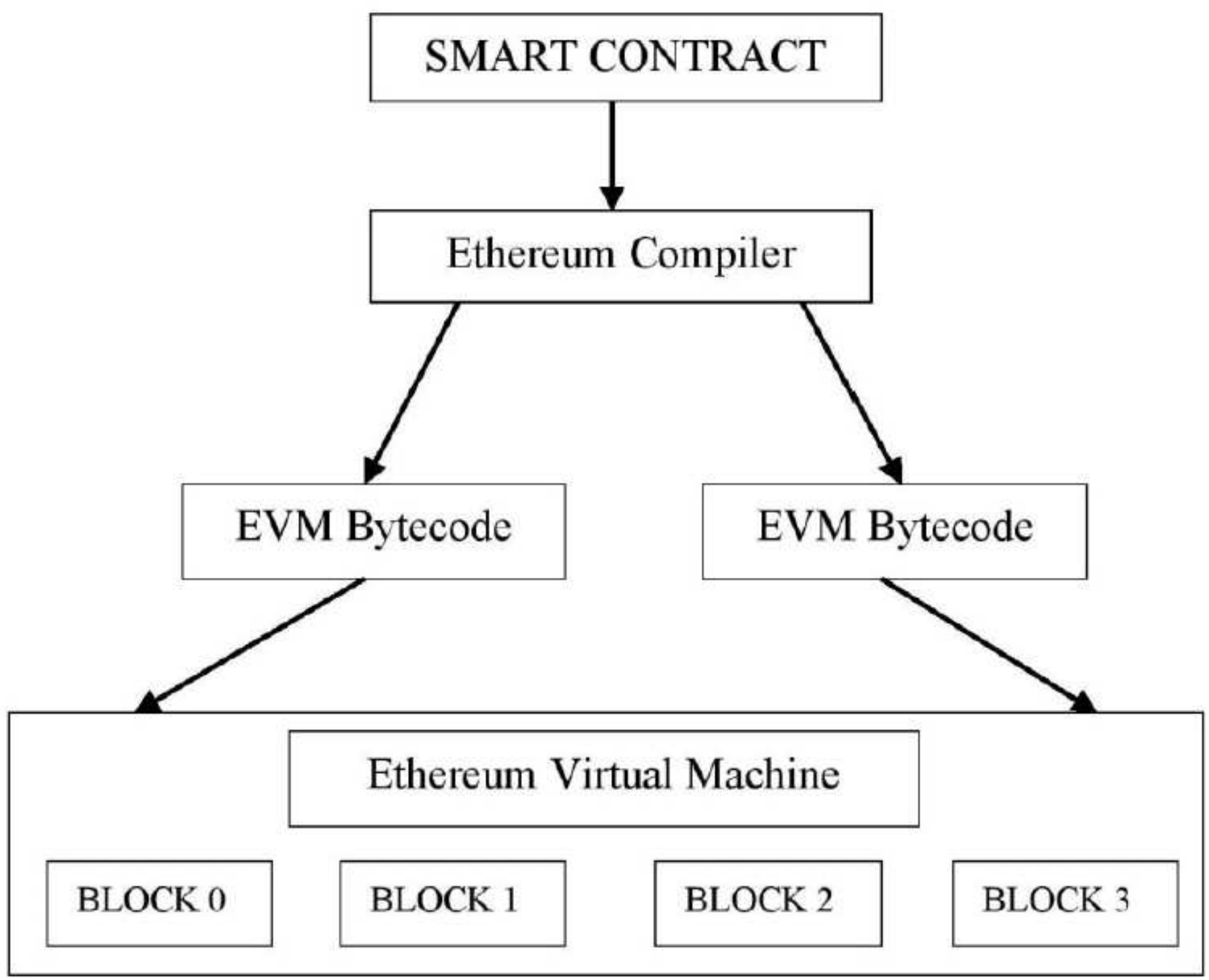

Figure 2

Smart contract execution 


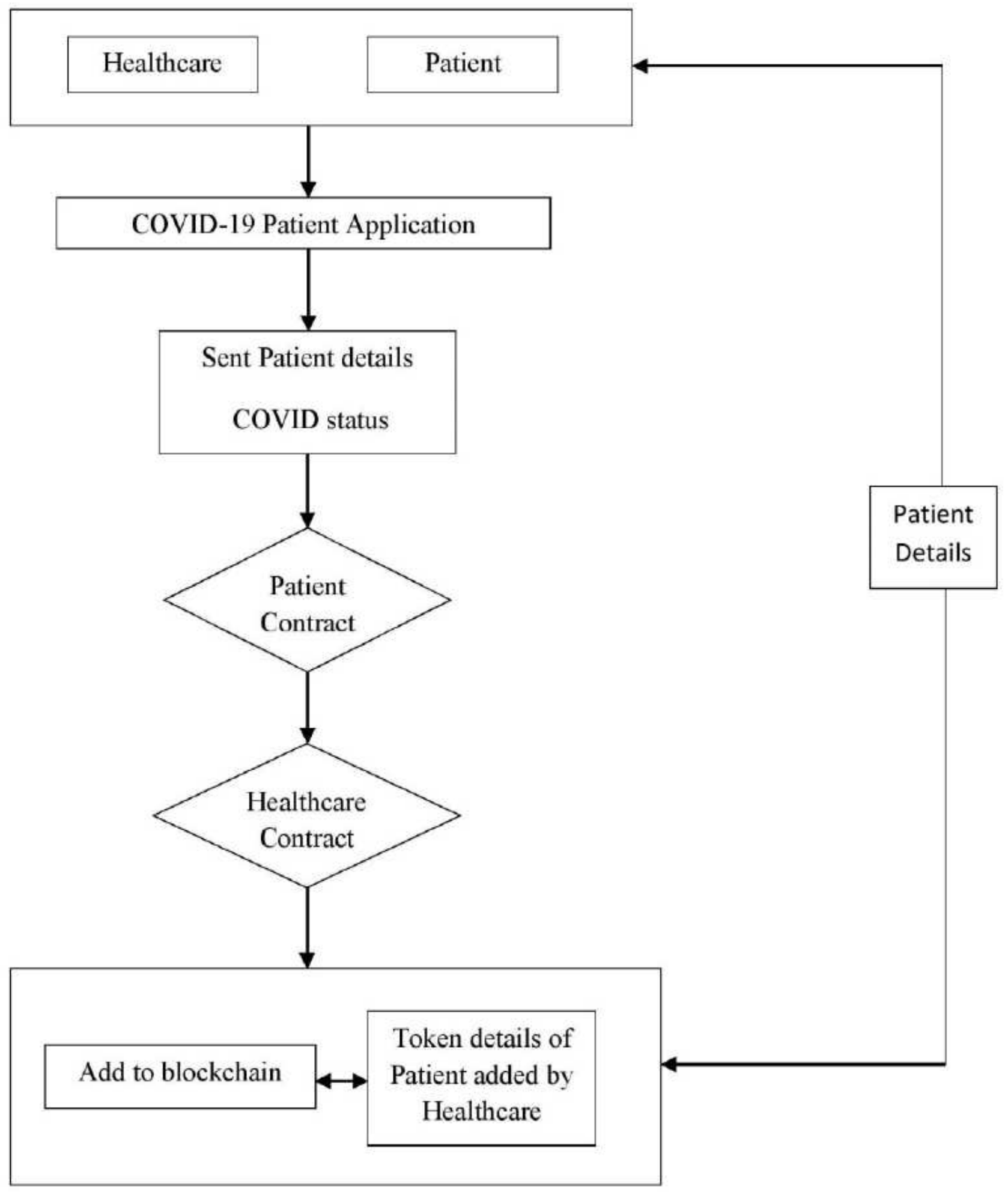

Figure 3

Proposed Blockchain workflow 


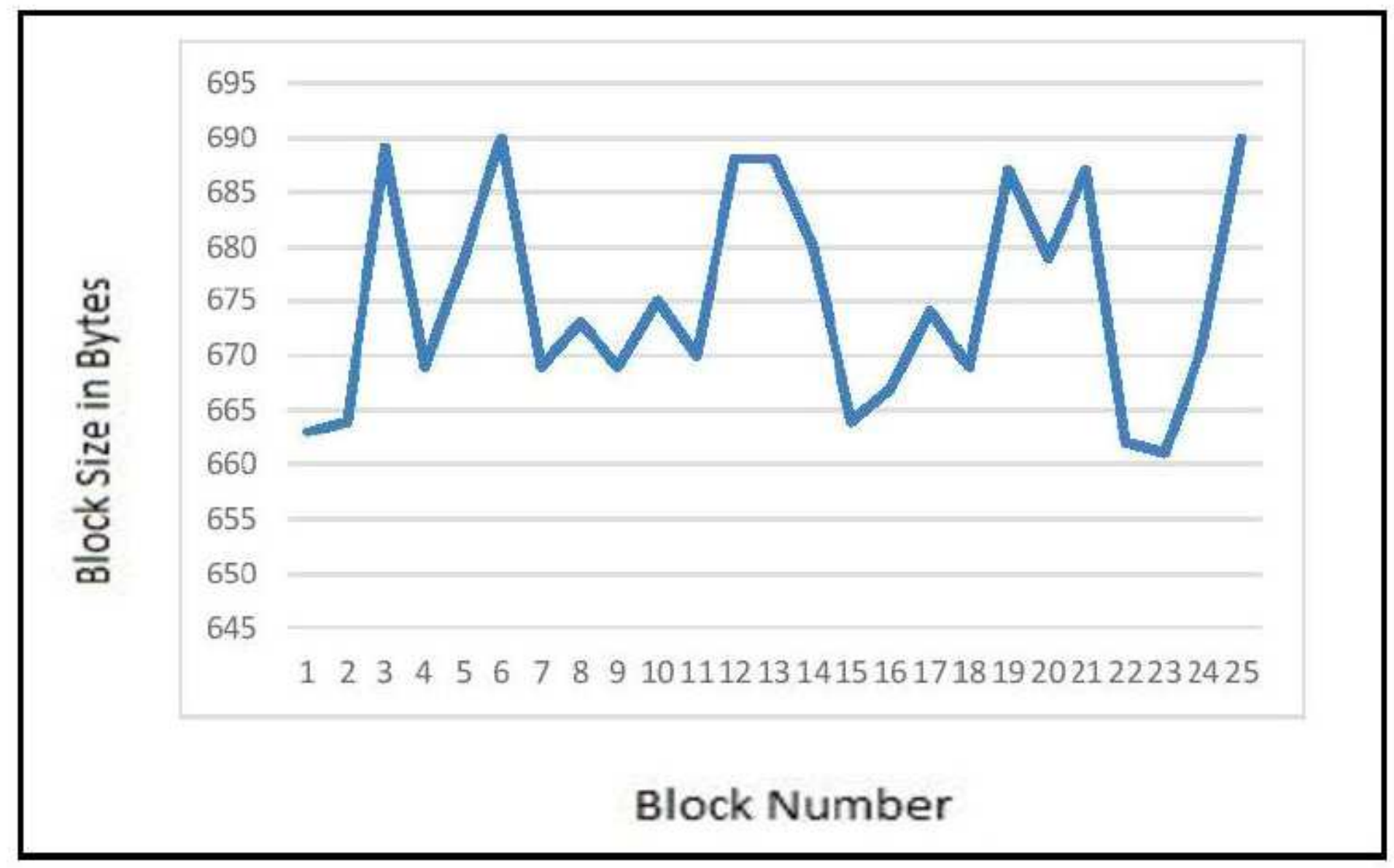

Figure 4

Proposed Blockchain workflow 


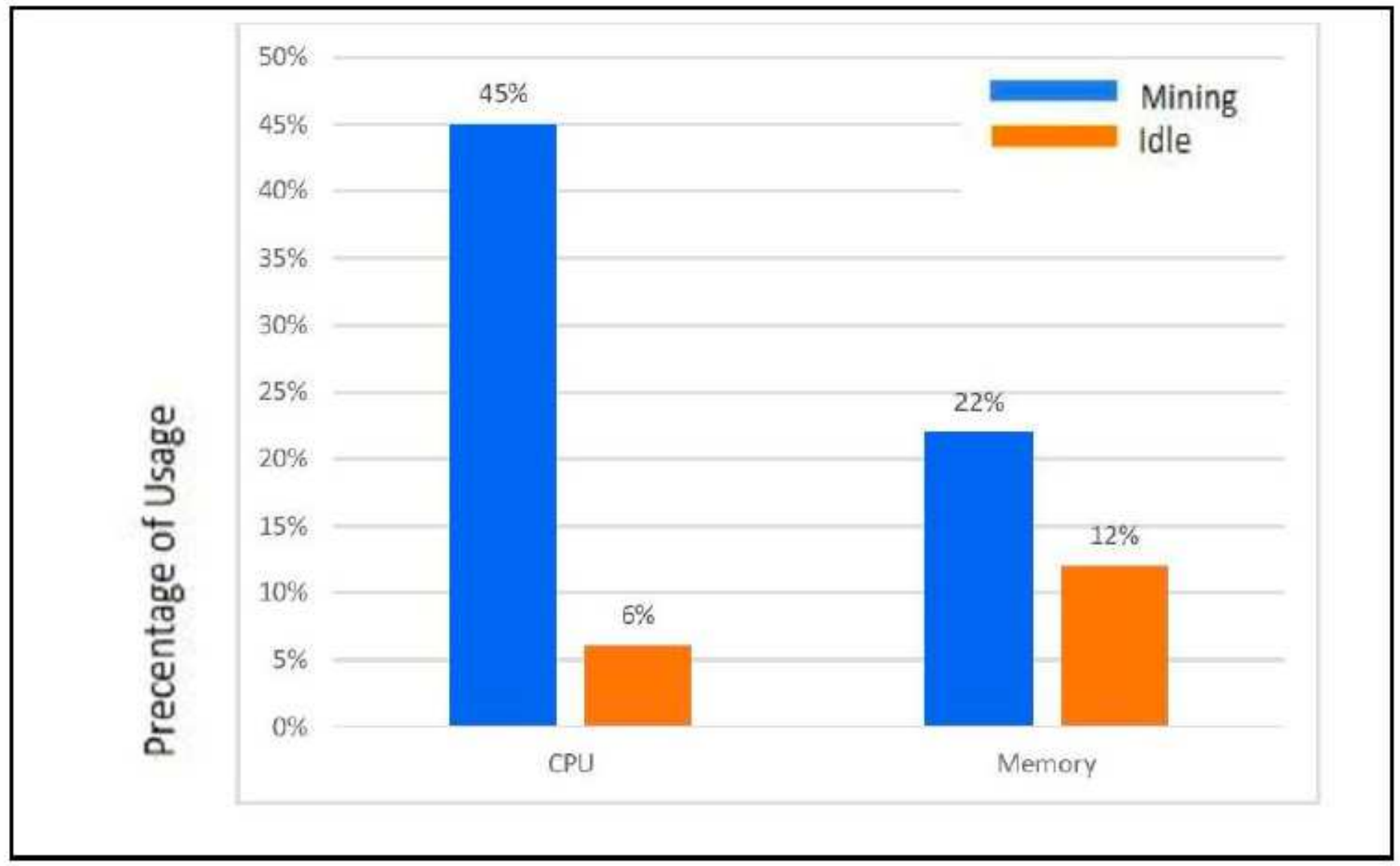

Figure 5

Block size 


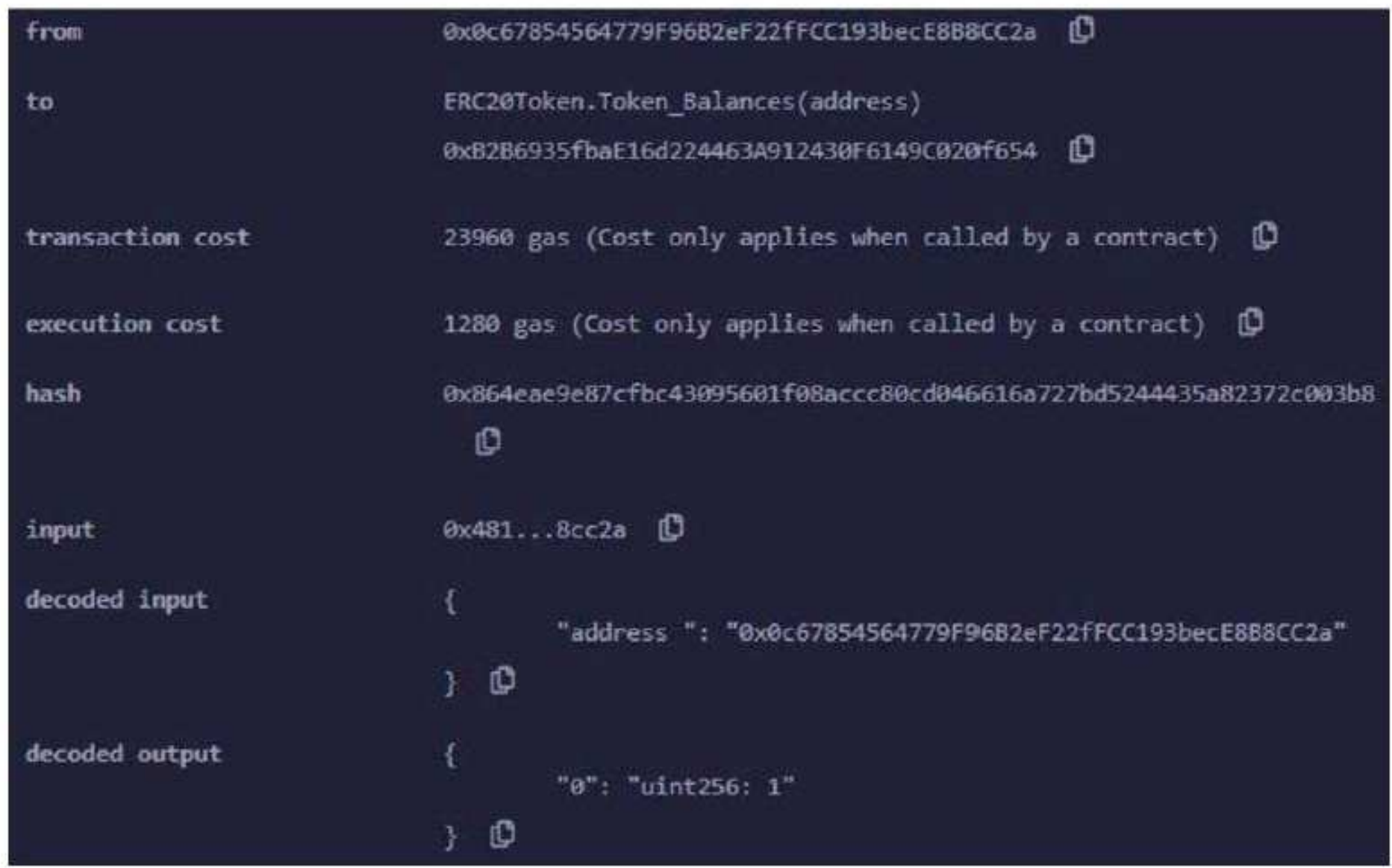

Figure 6

CPU and memory utilization comparison during mining and idleness 


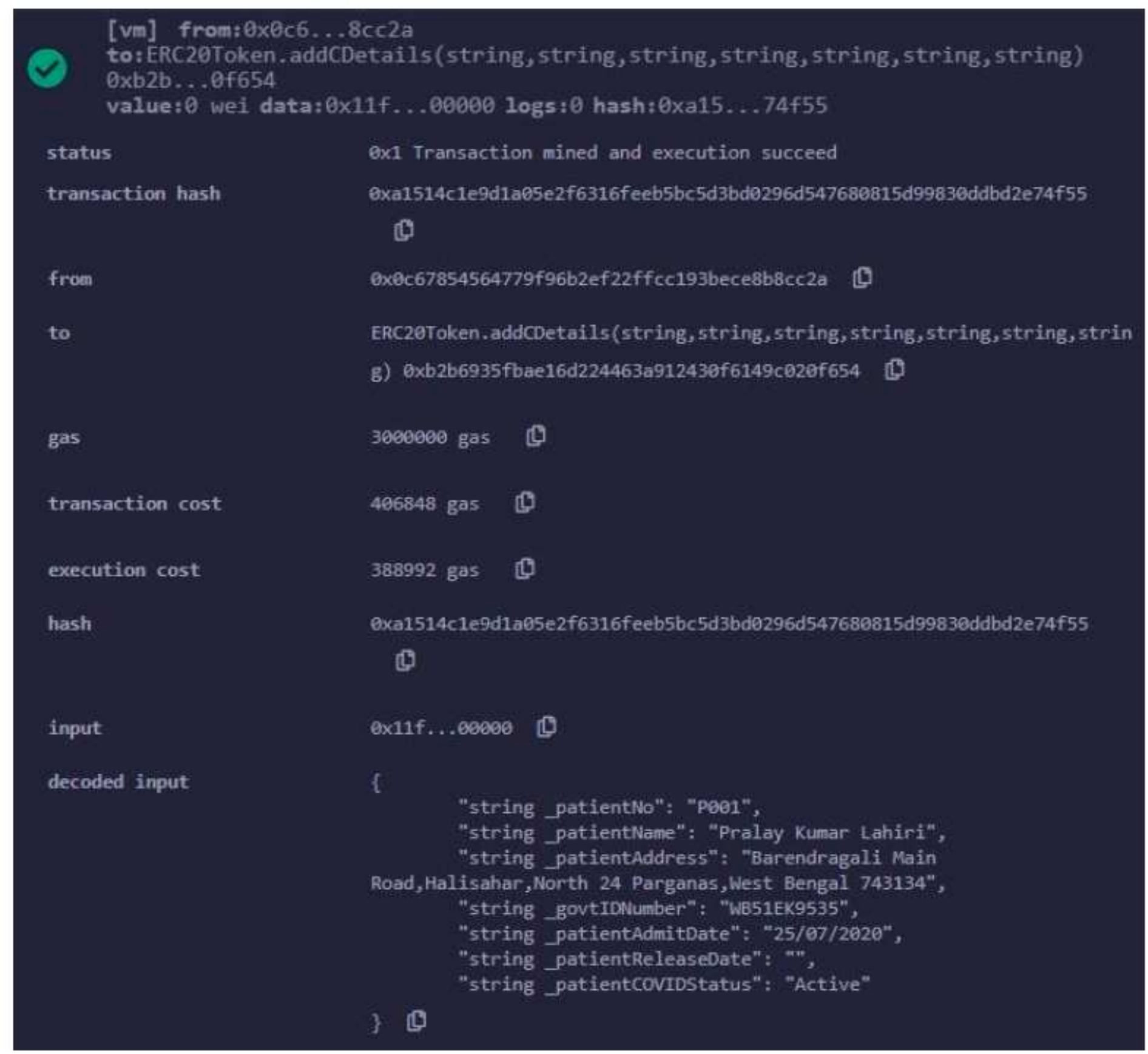

\section{Figure 7}

Token value passed by Healthcare 


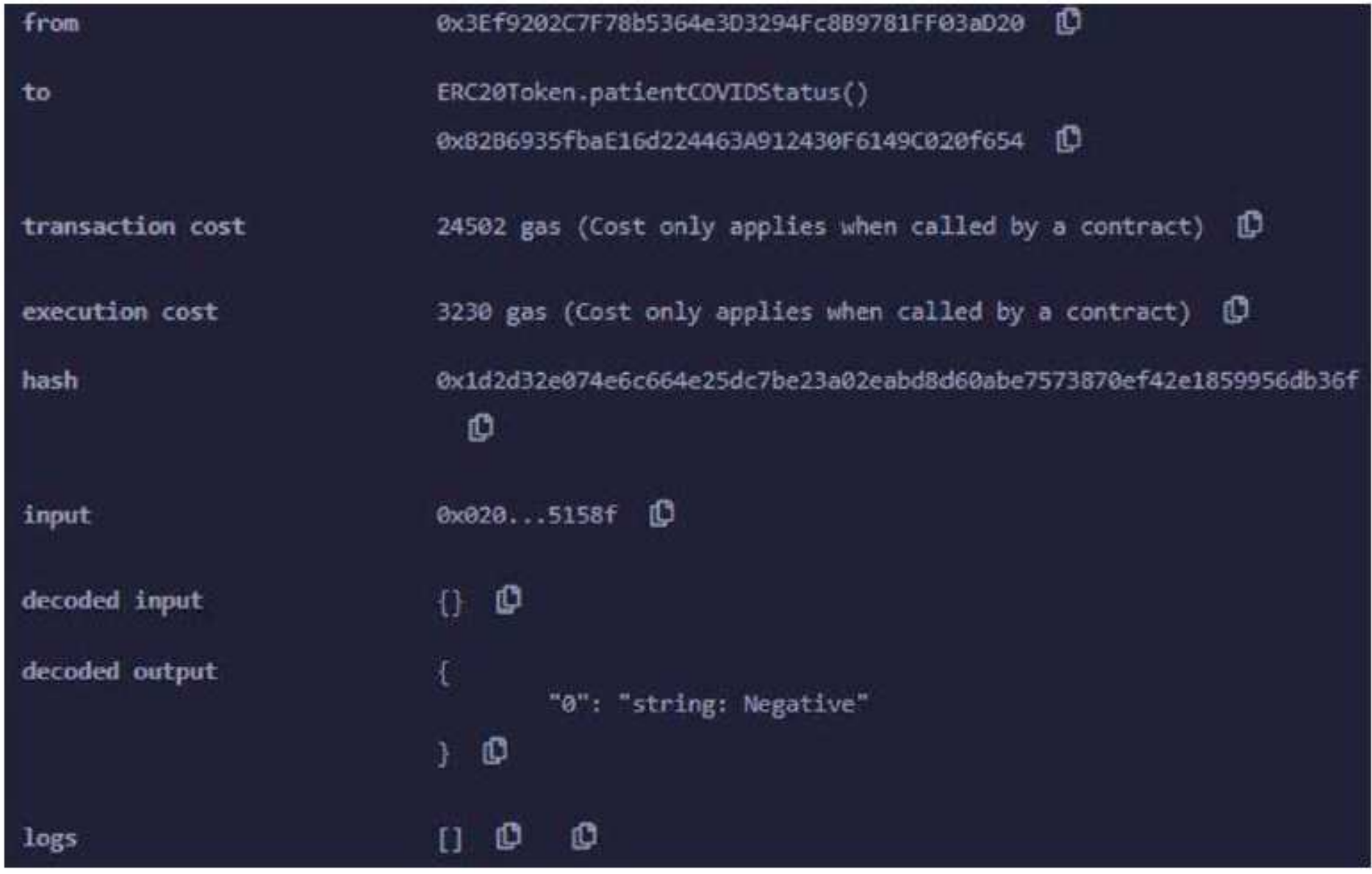

\section{Figure 8}

Patient data inserted successfully 


\begin{tabular}{|c|c|}
\hline from & exec67854564779f96b2ef22ffcc193bece8b8cc2a \\
\hline \multirow[t]{2}{*}{ to } & ERC20Token.addCDetails (string, string, string, string, string, string, strin \\
\hline & g) Exb2b6g35fbae16d224463a912430f $6149 \mathrm{co} 020 \mathrm{f} 654$ 마 \\
\hline gas & 3606000 gas b \\
\hline transaction cost & $406848 \mathrm{gas}$ \\
\hline execution cost & 388992 gas \\
\hline hash & $\begin{array}{l}\text { 6xae6532bab407d895b91b6b4211e82e661a088f ca70bc } 382232 \text { be } 563 \text { faa } 15 \text { be } 53 \\
\text { 해 }\end{array}$ \\
\hline input & $6 x e 55 \ldots 60000$ tb \\
\hline \multirow[t]{2}{*}{ decoded input } & "string ": "POO2" \\
\hline & ㄴ. ㄴ. \\
\hline \multirow[t]{3}{*}{ decoded output: } & ( \\
\hline & $\begin{array}{l}\text { Road, Hallsahar, North } 24 \text { Parganas, West Bengal } 743134 \text { ", } \\
\text { "3": "string: govtIDNumber WB51EK9536", } \\
\text { "4": "string: patientAdmitDate 25/07/2020", } \\
\text { "5": "string: patientReleaseDate", } \\
\text { "6": "string; patientCovIDStatus Active" }\end{array}$ \\
\hline & ]. 마. \\
\hline logs & [1] [ [ [ \\
\hline
\end{tabular}

\section{Figure 9}

Updated COVID status of patient 